\title{
Transient Epileptic Amnesia with Preserved Consciousness: a Report of Two Cases
}

Case Report

Journal of Epilepsy Research pISSN 2233-6249 / eISSN 2233-6257

Received February 15, 2017 Accepted February 27, 2017

Corresponding author: Jung-Ju Lee Department of Neurology, Eulji General Hospital, Eulji University College of Medicine, 68 Hangeulbisuk-ro, Nowon-gu, Seoul 01830, Korea

Tel. $+82-2-970-8312$

Fax. +82-2-974-7785

E-mail; jungju2@unitel.co.kr

\author{
Soohyun Cho, Wong-Woo Lee, Kyusik Kang, Jong-Moo Park, Byung-Kun Kim, Ohyun Kwon, \\ Jung-Ju Lee
}

Department of Neurology, Eulji General Hospital, Eulji University College of Medicine, Seoul, Korea

\begin{abstract}
Transient epileptic amnesia (TEA) is defined as recurrent ictal and interictal transient memory disturbances typically occurring upon waking. Patients with TEA usually cannot remember the episodes. Electroencephalographic (EEG) abnormalities are usually localized on the dominant or bilateral temporal lobes. Here we report two cases of TEA with atypical features. The patients could remember the episodes afterwards, and abnormal discharges on EEG were detected in the temporal lobes of the non-dominant hemisphere. We suggest that confinement of epileptic discharge to the non-dominant temporal lobe can lead to partial preservation of consciousness during seizure attacks that produce transient amnesia. (2017;7:54-56)
\end{abstract}

Key words: Transient amnesia, Epilepsy, Dominant temporal lobe

\section{Introduction}

Transient amnesia is a commonly encountered clinical manifestation with diverse causes. The most common cause of transient amnesia is transient global amnesia (TGA), which is characterized by the abrupt onset of anterograde amnesia without disturbances in other cognitive functions. TGA usually lasts for a few hours and is accompanied by typical behavioral changes. ${ }^{1,2}$ Transient epileptic amnesia (TEA) is a sub-type of mesial temporal lobe epilepsy characterized by recurrent episodes of transient memory disturbances. TEA closely resembles TGA because transient amnesia is sometimes the sole manifestation of the seizures. Although TEA is distinguished from TGA by a relatively brief duration (less than an hour), high frequency of episodes, common occurrence upon waking, patients with TEA generally cannot remember the amnestic episodes. ${ }^{2-5}$

Here we report the cases of two patients with TEA who presented with amnesia with preserved consciousness. They were amnesic during the episodes, but remembered the events after the episodes. These episodes were caused by non-dominant temporal lobe epilepsy. We will then discuss the mechanisms underlying our observations.

\section{Case}

\section{Case 1}

A 77-year-old right-handed man was admitted to our department with recurrent episodes of transient amnesia. The patient had no other notable past medical or psychiatric disease. Two days before admission, the patient experienced an episode of transient amnesia that began abruptly during breakfast. He could not remember his job and kept asking why he had to go to work despite his wife's repeated explanations. The symptoms lasted for about 10 minutes and then disappeared. Similar symptoms were observed again on the next day. The patient did not remember his nephew's wedding, which had taken place 4 days prior. He continued to ask when the nephew was married and why he was married so early. The patient's memories gradually returned over a period of 20 minutes. After these episodes, however, the patient remembered what happened during those events. During the above episodes, the patient's family did not observe any other cognitive dysfunction other than amnesia. The patient was alert, fluent, and not disoriented to place or person.

Routine physical and neurological examination revealed no abnormalities. The patient had a score of 29 on the Korean version of the mini-mental examination (K-MMSE) (2 out of 3 on memory recall test, could recall the other when cued). Magnetic resonance imaging (MRI) and MR angiography revealed no abnormalities in the hippo- 


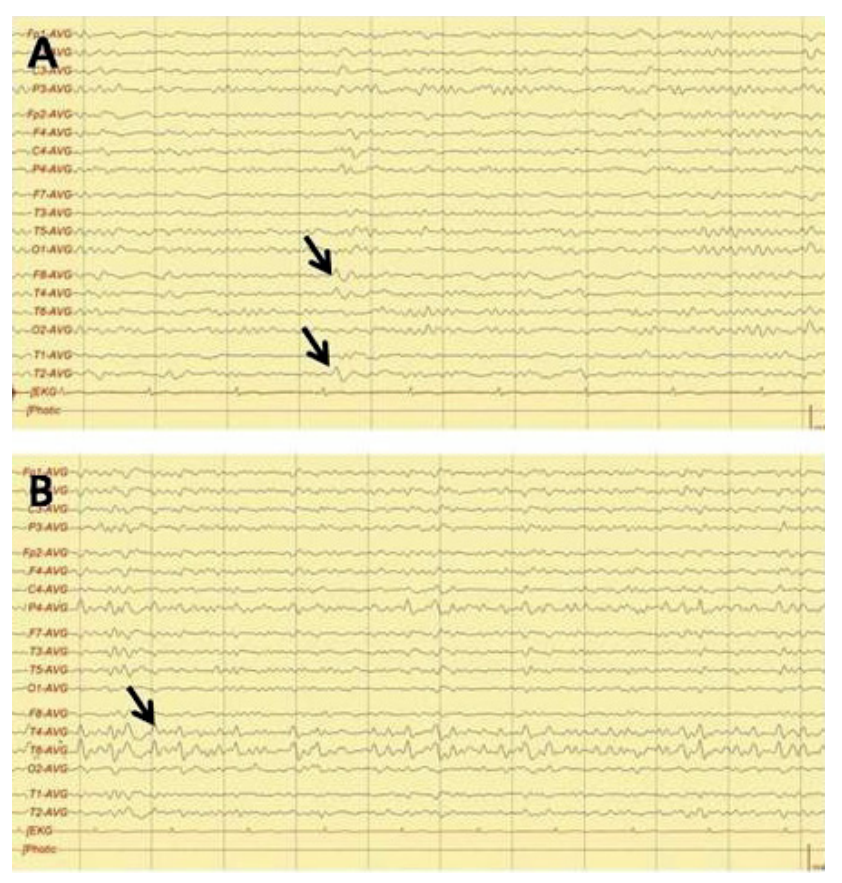

Figure 1. EEG findings of the patients. (A) EEG finding in Case 1. EEG indicatesthat the epileptiform discharges are evident in the right temporal leads, including F8 and T2 (arrows). (B) EEG finding in Case 2. EEG indicates intermittent rhythmic high-voltage activity and sharp waves in the right temporal area (arrow). EEG, electroencephalographic.

campi or other structures related to memory. On electroencephalographic (EEG), epileptiform discharges were observed in the right temporal leads, including F8 and T2 (Fig. 1A). Brain single-photon emission computed tomography revealed no significant increase or decrease of perfusion in the temporal lobes. Routine blood tests, including blood cell counts, routine chemistry tests, and tests to determine the levels of ammonia and creatine phosphokinase revealed no significant abnormalities. The patient was treated with $15 \mathrm{mg} / \mathrm{kg}$ of carbamazepine controlled-release (CR), followed by maintenance dose of $200 \mathrm{mg}$ twice per day. He has been free of memory disturbances for 3 months.

\section{Case 2}

A 63-year-old right-handed woman was admitted for evaluation of recurrent and brief memory disturbances. Her husband noticed that the patient suffered from retrograde amnesia upon waking. Specifically, the patient repeatedly asked about what she did last night. The amnesia lasted for about thirty minutes. During these attacks, cognitive functions other than memory seemed to remain intact. One month later, when the patient exited the subway train, she lost her way for about 10 minutes. The patient remembered these amnesic episodes and what she did during those events.

Routine physical and neurological examination revealed no abnormalities. The patient had a score of 24 on the K-MMSE (1 out of 5 on the attenuation and concentration test and 1 out of 3 on the memory recall test, could recall the others when cued). The patient did not show significant declines in cognitive functions and activities of daily living observed by her family. Brain MRI, MR angiogram, and EEG revealed no abnormalities. Routine blood tests also revealed no abnormalities. The patient was treated with $300 \mathrm{mg}$ of valproic acid twice per day. She had not been taking any medicine about cognitive dysfunction before and after those tests. Her symptoms did not recur for 3 years.

However, she developed transient repeated amnesia after the symptom-free interval. Diffusion-weighted MRI and blood tests revealed no abnormalities, but intermittent rhythmic high-voltage sharp waves were observed in the right temporal area (Fig. 1B). After the addition of $100 \mathrm{mg}$ zonisamide to her medication, the patient has been symptom-free for 1 month.

\section{Discussion}

TEA is a rare but distinct temporal lobe epilepsy, and is characterized by recurrent ictal transient memory disturbances. These disturbances typically occur upon waking. TEA is often accompanied by other temporal seizures, such as olfactory hallucinations and oral automatisms. It responds favorably to anti-epileptic medication. ${ }^{3-5}$ Until now, the reported EEG findings have mostly been interictal. These findings include unilateral or bilateral temporal sharp waves, nonspecific focal slow waves, or are normal. ${ }^{6}$ EEG abnormalities areusually observed in the dominant side or in bilateral areas.

Our patients had many features compatible with the diagnostic criteria proposed by Zeman and Bulter. ${ }^{4}$ Specifically, they had histories of recurrent episodes of transient amnesia lasting for less than an hour and responded well to anti-epileptic medication. In addition, their cognitive abilities, such as perception and language, were intact during the episodes. ${ }^{4}$

TEA is easily mistaken for TGA or psychogenic amnesia. However, episodes of TGA tend to last several hours and have lower recurrence rates, while episodic memory of psychogenic amnesia remains intact and the acquisition for new memories is preserved. ${ }^{1,2,7}$ Thus, both of these conditions differ from those seen in our patients.

Our patients had atypical clinical and EEG findings. Specifically, they remembered the amnesic episodes. Few patients with TEA can 
remember their attacks in detail. ${ }^{6}$ Interestingly, these patients displayed repetitive questioning during the TEA attacks. Another interesting observation was that abnormal discharges on EEG were detected on the temporal lobe of the right hemisphere (speech/non-dominant side), but not on the dominant side or bilaterally.

It has been reported that the lateralization of temporal lobe seizures may be the predominant feature determining whether consciousness is preserved ictally. The dominant hemisphere is primarily responsible for the conscious state. ${ }^{8-10}$ Consciousness impairments are more often observed in patients with dominant temporal seizures than in patients with seizures in non-dominant areas. ${ }^{11}$ In addition, several reports indicate that consciousness is preserved at seizure onset as long as synchronization remains confined to the non-dominant temporal lobe. Loss of consciousness is thought to be directly related to excessive synchronization of neuronal activity in extra-temporal lobe structures, including the thalamus and parietal cortices. ${ }^{12-14} \mathrm{We}$ thus hypothesize our patients were able to recollect the amnestic episode itself after the episode because ictal rhythms originating from the non-dominant temporal lobe did not propagate to extra-temporal structures, such as the thalamus and associated cortices, which are involved in loss of consciousness during the attack.

In summary, we report two cases of TEA with atypical features, including recollection of the amnestic episode and abnormal discharges in the non-dominant temporal lobe. We propose that confinement of epileptic discharge to the non-dominant temporal lobe can lead to partial preservation of consciousness during seizure attacks that produce transient amnesia.

\section{References}

1. Owen D, Paranandi B, Sivakumar R, Seevaratnam M. Classical dis- eases revisited: transient global amnesia. Postgrad Med J 2007:83:236-9.

2. Nicastro N, Picard F, Assal F. Transient global amnesia mimics: transient epileptic amnesia. Epilepsy Behav Case Rep 2014;2:100-1.

3. Bilo L, Meo R, Ruosi P, De Leva MF, Striano S. Transient epileptic amnesia: an emerging late-onset epileptic syndrome. Epilepsia 2009;50 Suppl 5:58-61.

4. Zeman A, Butler C. Transient epileptic amnesia. Curr Opin Neurol 2010;23:610-6.

5. Asadi-Pooya AA. Transient epileptic amnesia: a concise review. Epilepsy Behav 2014;31:243-5.

6. Butler C, Zeman A. Recent insights into the impairment of memory in epilepsy: transient epileptic amnesia, accelerated long-term forgetting and remote memory impairment. Brain 2008;131(Pt 9):2243-63.

7. Serra L, Fadda L, Buccione I, Caltagirone C, Carlesimo GA. Psychogenic and organic amnesia. A multidimensional assessment of clinical, neuroradiological, neuropsychological and psychopathological features. Behav Neurol 2007;18:53-64.

8. Albert ML, Silverberg R, Reches A, Berman M. Cerebral dominance for consciousness. Arch Neurol 1976;33:453-4.

9. Hoffmann JM, Elger CE, Kleefuss-Lie AA. Lateralizing value of behavioral arrest in patients with temporal lobe epilepsy. Epilepsy Behav 2008;13:634-6.

10. Englot DJ, Blumenfeld $H$. Consciousness and epilepsy: why are complex-partial seizures complex? Prog Brain Res 2009;177:147-70.

11. Lux S, Kurthen M, Helmstaedter C, Hartje W, Reuber M, Elger C. The localizing value of ictal consciousness and its constituent functions. Brain 2002;125:2691-8.

12. Lee $K$, Meador $K$, Park YD, et al. Pathophysiology of altered consciousness during seizures subtraction SPECT study. Neurology 2002:59:841-6.

13. Blumenfeld $H$, McNally KA, Vanderhill SD, et al. Positive and negative network correlations in temporal lobe epilepsy. Cereb Cortex 2004; 14:892-902.

14. Arthuis $M$, Valton L, Regis J, et al. Impaired consciousness during temporal lobe seizures is related to increased long-distance corticalsubcortical synchronization. Brain 2009;132:2091-101. 